\title{
Reduced-Order Models for Representing Converters in Power System Studies
}

\author{
Yunjie Gu, Nathaniel Bottrell, and Timothy C. Green, Senior Member, IEEE
}

\begin{abstract}
A reduced-order model that preserves physical meaning is important for generating insight in large-scale power system studies. The conventional model-order reduction for a multiple-timescale system is based on discarding states with fast (short-timescale) dynamics. It has been successfully applied to synchronous machines, but is inaccurate when applied to power converters because the timescales of fast and slow states are not sufficiently separated. In the method proposed here, several fast states are at first discarded but a representation of their interaction with the slow states is added back. Recognizing that the fast states of many converters are linear allows well-developed linear system theories to be used to implement this concept. All the information of the original system relevant to system-wide dynamics, including non-linearity, is preserved, which facilitates judgments on system stability and insight into control design. The method is tested on a converter-supplied mini power system and the comparison of analytical and experiment results confirms high preciseness in a broad range of conditions.
\end{abstract}

Index Terms-Power Converter, Model-Order Reduction, Multiple-Scale, Power System Stability

\section{NOMENCLATURE}

$v_{o}, i_{o}$ Output voltage and current at point of connection

$v_{f}, i_{f}$ Filter capacitor voltage and inductor current

$v_{c} \quad$ Converter modulated voltage

$v_{d c} \quad$ DC-link voltage

$i_{i} \quad$ Integrator current

$p, q \quad$ Active and reactive power

$p_{d c} \quad$ DC source power

$\omega_{1} \quad$ Common frequency

$\epsilon \quad$ Estimated phase angle error

$\omega, \delta \quad$ Internal frequency and phase angle

$k_{p}, k_{i} \quad$ Current loop proportional/integral gain

$h_{p}, h_{i} \quad$ Voltage loop proportional/integral gain

$a_{p}, a_{i}$ Active power (DC) loop proportional/integral gain

$b_{p}, b_{i} \quad$ Reactive power loop proportional/integral gain

$\kappa_{p}, \kappa_{i}$ Phase-locked loop proportional/integral gain

$L_{o} \quad$ Output Inductor

$L_{f}, C_{f}$ Filter inductor and capacitor

$R_{v}, X_{v}$ Virtual resistance and reactance

$D_{\omega}, J_{\omega}$ Frequency droop coefficient and inertia

$\lambda_{n}, \lambda_{n}^{\prime}$ The $n$th eigenvalue of the full and reduced model

$e_{n} \quad$ Relative error between $\lambda_{n}$ and $\lambda_{n}^{\prime}$

Yunjie Gu, Nathaniel Bottrell and Timothy C. Green are with the Department of Electrical and Electronic Engineering, Imperial College, London. E-mail: yunjie.gu@imperial.ac.uk; nathaniel.bottrell04@imperial.ac.uk; t.green@imperial.ac.uk.

This work was sponsored by EPSRC project Resilient Hybrid Technology for High-Value Microgrids (RHYTHM), EP/N034570/1.

The model data and Matlab files used in generating the results in this paper are available from the website https://spiral.imperial.ac.uk. $f_{s} \quad$ Switching frequency

* Superscript for controller reference value

- Superscript for complex conjugation

$d q \quad$ Subscript for internal rotating reference frame

$D Q \quad$ Subscript for common rotating reference frame

\section{INTRODUCTION}

Power converters are becoming increasingly common as interfaces for energy resources and loads and appearing at higher power ratings such that they become an important feature of the dynamics of a power system. In some cases, e.g. wind/solar farms and microgrids, they even dominate a grid locally [1], where severe stability problems have been reported [2]-[7]. Effective mathematical representation of converters is fundamental for system-wide stability studies. Impedance (or admittance) models have long been used to this end, but are limited to only small-signal analysis. Moreover, such models compress the whole network into a single impedance ratio, so they are not interpretive in multi-machine oscillations nor in relating problems back to physical features [8]-[13].

On the other hand, state-space models provide a general tool in which the representation of the dynamics is rich in detail [14], [15]. Both small-signal and large-signal models can be formulated, and participation and sensitivity analysis allows for insight into multi-machine interactions. Unfortunately, they are not widely accepted so far in converter dominated systems, mainly because of their formidable complexity. A converter itself may have tens of states, and hundreds of converters may be present in a local network (e.g., in a wind farm) and so the model order becomes immense. What makes things worse is that these systems have a wide timescale, ranging from milliseconds to seconds, which means that in numerical simulation a small time-step is required with a broad time horizon so the computational effort becomes very large.

To pave the way for easier application of state-space models, appropriate model-order reduction (or model reduction, for short) is needed. The classical approach is based on multiplescale analysis [16], which asserts that the fast (short-timescale) and slow (long-timescale) dynamics of a system are almost independent provided that their timescales are sufficiently separated. In the light of this principle, the state variables of a multiple-scale system can be grouped into those that participate in the fast and in the slow dynamics. The fast states can be removed (by replacing the corresponding state equation with a pure gain) when evaluating slow dynamics whereas the slow states can be held stationary when evaluating fast dynamics. This yields reduced models with lower order and 
narrower timescale than the full model [17]. In large-system studies, slow dynamics normally have higher impact and wider propagation than fast dynamics, and so are of most interest.

The fast and slow states can be identified by eigenvalues. For linear systems, fast states are defined as those associated with large eigenvalues, and slow states by small eigenvalues. For nonlinear systems, eigenvalues are not explicit globally, but can be approximated by averaging all locally linearized systems along possible trajectories, which also gives the criterion for separating states in timescale [18].

Multiple-scale based model reduction has a successful application in conventional power systems with synchronous machines, where fast electric-magnetic states are neglected in slow electric-mechanical transient analysis [19]. It is a natural idea to seek an extension to power converters. However, in power converter design, the speeds of fast states are bounded from above due to the limitation of switching frequency and filters, whereas the speeds of slow states are bounded from below because of the absence of mechanical inertia. Combined, these factors mean that the fast and slow states may actually be quite close together in timescale and interaction between them can be significant. This is especially true when converter control parameters are pushed to extremes, and simply removing fast states may cause unacceptable modelling error and even lead to a misjudgment on stability.

The poor separation of fast and slow states can be overcome by preserving a portion of dynamics associated with the fast states in the reduced model, so that the interaction of the fast states with the slow states is represented rather than truncated. But the problem is how to identify and quantify this interactive portion. Fortunately, the fast states of most converters are linear (considering that the switching-ripple nonlinearity is washed out by switching-cycle averaging) [20]. This feature enables us to leverage well-developed linear system theories to analysis the structure of the fast sub-model, identify the dominant modes with which the slow states may overlap in timescale, and add them back to the reduced model. It ensures a reasonable selection of the dynamics to be truncated, so as to preserve the essential information (including non-linearity of the slow states) of the original system.

With these considerations in mind, we develop a systematic model reduction method, which proves to be a useful tool for system-wide stability analysis and design. The paper is organized as below. Section II gives a general description for the methodology proposed for model reduction and then in Section III reduced state-space models for typical converters are derived. These individual converter models are interconnected in a mini power system to demonstrate the effectiveness of the models in system-wide stability analysis in Section IV, with experiment verification provided in Section V. The last section concludes this paper.

\section{Model Reduction Methodology}

A power network with multiple power converters is a system-of-systems and as such we have two routes for model reduction, as illustrated in Fig. 1. The first is to build a model of the entire system and reduce it collectively whereas the second is to reduce each converter model individually before linking them into a system. The first is more accurate since it retains all the details of the interconnected system before reduction. On the other hand, the second has the advantage of better scalability and lower computational complexity. This paper is focused on the second approach but with special attention paid where interconnection may induce new modes.

The converters in power systems share common features which lay the basis for our model reduction concept. It is shown in [21] that the eigenvalues of converters in power systems can be grouped into distinct clusters, among which the fast (high-frequency) ones are related to inner control loops and harmonic filters, whereas slow (low-frequency) ones are related to outer control loops and dc-link capacitors. The clustering of eigenvalues facilitates the separation of states and the division of the complete converter model into the fast and slow sub-models, as shown in Fig. 2.

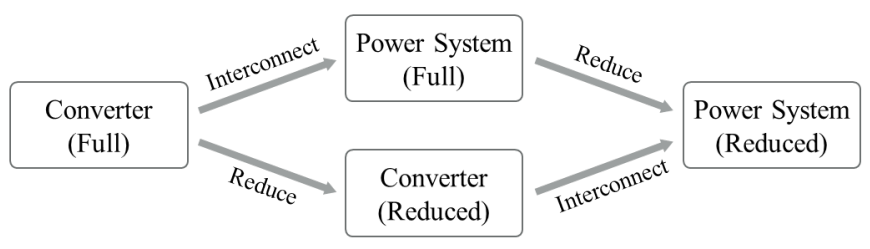

Fig. 1. Centralized and decentralized model reduction

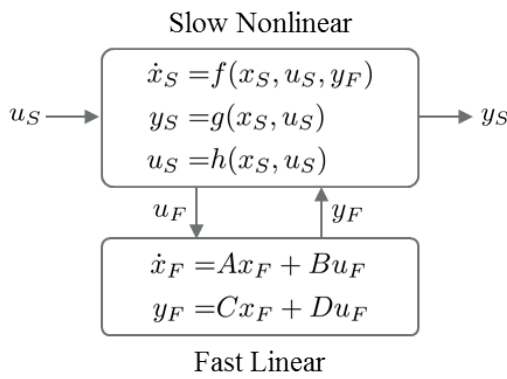

Fig. 2. General structure for converter models.

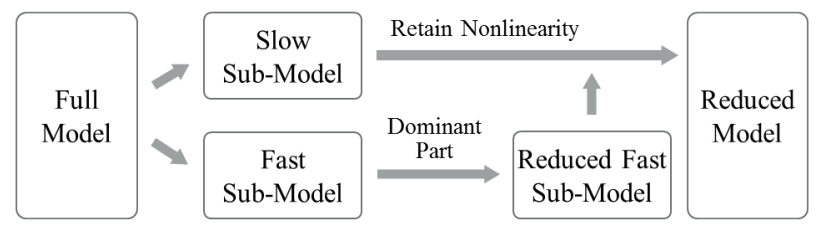

Fig. 3. Model reduction with "peel-off and add-back".

The fast sub-model is almost linear, because it is basically composed of linear circuits and linear controllers. This linearity enables a selective truncation of fast dynamics without distorting the slow ones, which leads to the methodology in Fig. 3. The essential process can be described as "peel-off and add-back": we peel off all the fast states initially, and add back a representation of the dominant part to restore accuracy. Several algorithms are available for extracting the dominant part from a linear system, including balanced truncation, 
moment matching, modal truncation, and so on [22], [23]. These methods set different targets to be optimized, either in terms of energy, observability, or frequency response. The latter one is used here, because our methodology is rooted in the separation of timescales, which is closely related to frequency. In conventional frequency-based model reduction methods (e.g., modal truncation), only poles are of concern. However, considering that zeros may become poles when the inputs and outputs are reversed, zeros may be of importance as well. Therefore, we take the approach of pole-zero truncation.

In this method, a fast sub-model is represented as a transfer matrix $G$ in pole-zero form

$$
G_{m n}(s)=\prod\left(s-z_{l}\right) / \prod\left(s-p_{k}\right) .
$$

To extract the dominant part of $G$, we preserve the poles and zeros near the static operating point, but truncate the others. If the model is formulated in the synchronous frame, the operating point is dc, so the preserved region in the complex plane is a circle centred on the origin (representing dc) with a radius $\omega_{c}$ representing the critical frequency, as illustrated in Fig. 4. If the model is formulated in the stationary frame, the operating point becomes ac, and the preserved region should be centred on $\pm j \omega_{s}$ accordingly. We use synchronous frame model in this paper, so the preserved region is $|s|<\omega_{c}$ and the dominant transfer function $G^{\prime}$ can be written as

$$
G_{m n}^{\prime}(s)=\frac{\prod_{\left|z_{l}\right|<\omega_{c}}\left(s-z_{l}\right)}{\prod_{\left|p_{k}\right|<\omega_{c}}\left(s-p_{k}\right)} \cdot \frac{\prod_{\left|z_{l}\right| \geq \omega_{c}} z_{l}}{\prod_{\left|p_{k}\right| \geq \omega_{c}} p_{k}} .
$$

Equation (2) can be rewritten in state-space format via minimum realization [24], and be added back into the reduced model. The selection of critical frequency $\omega_{c}$ is dependent on the bandwidth of slow dynamics. Generally, they are designed to be lower than the synchronous frequency $\omega_{s}$ to reject disturbances caused by unbalance and offset. Therefore, $\omega_{c}=\rho \omega_{s}$ is a reasonable choice, in which $\rho$ is a coefficient slightly higher than 1 for extra safety margin.

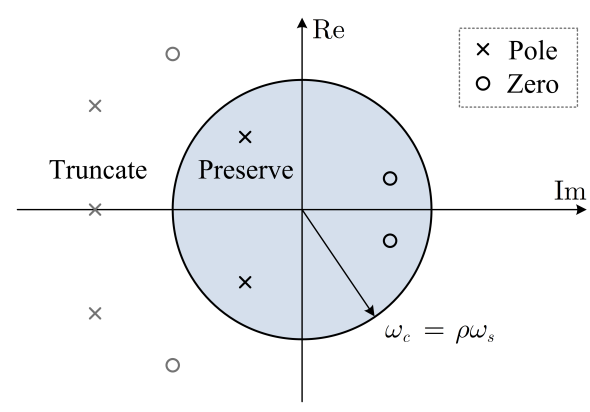

Fig. 4. Extracting dominant model by pole-zero truncation.

\section{TYPICAL CONVERTERS}

We proceed with applying our method on typical converters. There are various types of converter deployed in power systems. Current source converters (CSCs) or line-commutated converters (LCCs) have been used for high voltage dc (HVDC) transmission for half a century, whose standard model are well established. Voltage source converters (VSCs) are more recent but now more numerous. VSCs themselves show many variations, ranging from two-level to multi-level, and from single-phase to three-phase. For this study, the difference in the number of levels is not of concern provided that the effective switching frequency is high enough that statespace averaging gives a good two-level equivalence for multilevel converters. Single-phase systems present an additional complication, namely, an ac operating point of the converter since it has to be modelled in the stationary frame. This can be addressed by shifting the centre of the preserved region in pole-zero truncation to $\pm j \omega_{s}$ (representing $50 \mathrm{~Hz}$ operating point), and by using linear time-periodic (LTP) not linear timeinvariant (LTI) theories in subsequent small-signal analysis [25]. On the other hand, single-phase converters have much lower power rating than three-phase ones, and are of lower influence on power system stability [26]-[28]. Therefore, we focus on two-level three-phase VSCs herein, whose topology is shown in Fig. 5.

In spite of their common topology, VSCs may have different models due to different control patterns, which give rise to the distinction between current controlled VSC (CVSC) and voltage controlled VSC (VVSC) [29]. The former type is also called grid-feeding VSC since it can only feed current/power to an external voltage source and cannot work independently. The latter one is also known as grid-forming or grid-supporting VSC, since it can operate independently as a voltage source. The difference between forming and supporting is that gridforming ones are, ideally, stiff in terms of voltage or frequency, and cannot be connected in parallel, whereas a grid-supporting VSC is softer and suitable for parallel operation. A gridforming VSC can be treated as a special case of a gridsupporting one with droop coefficients of zero.

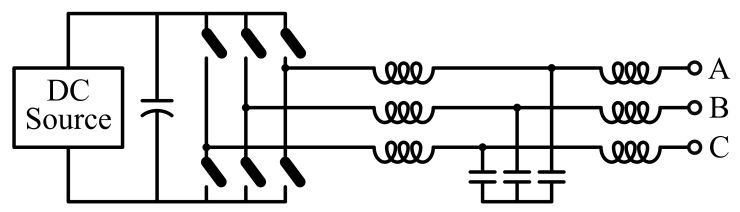

Fig. 5. Two-level three-phase VSC topology.

\section{A. CVSC}

In line with the general structure in Fig. 2, the model for a CVSC is displayed in Fig. 6. The slow sub-model sends current reference $i_{d q}^{*}$ and grid voltage $v_{o d q}$ to the fast submodel, which feeds back capacitor/converter voltage $\left(v_{f d q}\right.$, $\left.v_{c d q}\right)$ and grid/inductor current $\left(i_{o d q}, i_{f d q}\right)$ for phase-locked loop and power control loop. The fast sub-model contains eight states representing the current control loop and LCL filter on two axes. The time delay in digital controller or PWM channel can also be incorporated in the fast sub-model, but the time delay is on the scale of a switching cycle and has minor influence on slow dynamics, and is therefore neglected. There may be some other variants but the overall structures are similar. The model is formulated in the internal (local) rotating frame $(d q)$, but port variables are transformed to the common synchronous frame $(D Q)$ for interconnection with other converters, and the phase-locking and swing dynamics are reflected by the angle difference between $d q$ and $D Q$ 


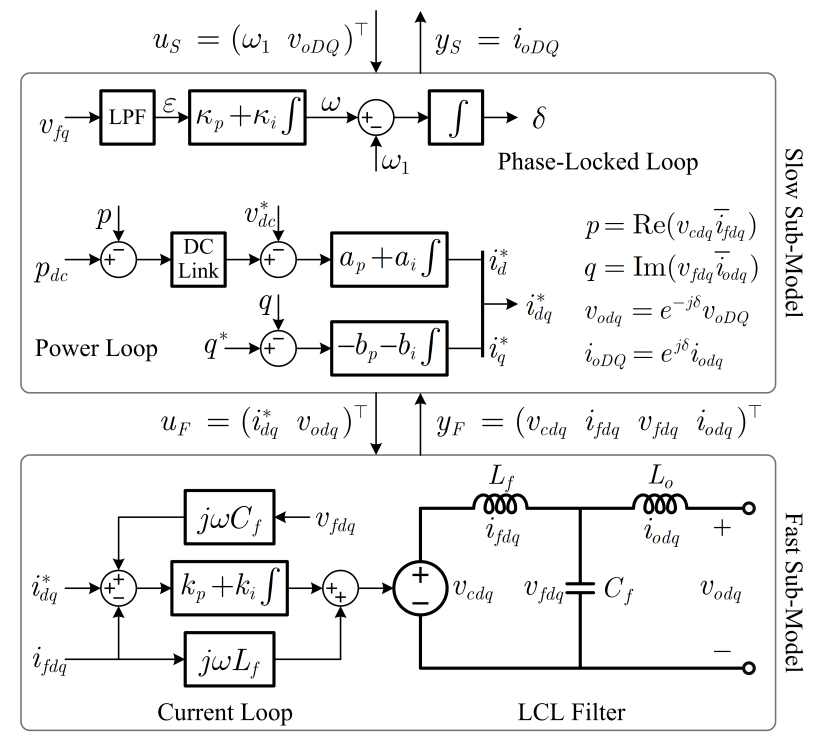

Fig. 6. CVSC model including phase-locked loop, power loop, current loop, and LCL filter.

frames. This is a common practice for modeling multi-machine ac systems [30]. To make the equation more concise, we use complex signals to combine the $d q(D Q)$ axis variables in a single variable as

$$
x_{d q}=x_{d}+j x_{q}, x_{D Q}=x_{D}+j x_{Q} .
$$

Since the location of poles and zeros are dependent on parameters, we need to identify the parameter values before model reduction is attempted. A standard procedure is available for choosing VSC parameters with the target of achieving a reasonable allocation of bandwidth among different control loops: the current and voltage loops are allocated a high bandwidth, above $\omega_{s}$, for fast reaction, whereas the phaselocked and power loops are allocated a low bandwidth, below $\omega_{s}$, for noise rejection. The detailed procedure can be found in [21] and [30]. Following this procedure, a set of typical parameters, as listed in Table I, were chosen. The parameters are presented in per-unit (pu) for scalability using the base values listed in the appendix.

Now we extract the dominant part from the fast sub-model. Following the principles in Section II, we calculate the transfer function matrix from $u_{F}$ to $y_{F}$, and list the dominant poles and zeros in Table II. All pole-zero values are normalized to the frequency of the internal rotating frame $\omega$. It should be noted that $\omega$ may change in time which makes the $d q$ frame model time-varying. However, $\omega$ is usually kept within a narrow range (e.g. $\pm 1 \%$ around $\omega_{s}$ ), and varies much more slowly than the fast states. Therefore, it makes sense to consider $\omega$ as quasi-constant.

The numeric expression in Table II may lose generality in case of parameter changes, but we manage to get a symbolic expression which holds for different of parameters, based on which a symbolic transfer function matrix is derived, which is displayed in Table III. The detailed calculation is presented in the appendix for the sake of brevity. From these transfer functions, we may track the origin of the dominant poles and zeros. The zeros from $i_{d q}^{*}$ to $v_{c d q}$ and $v_{f d q}$ is caused by the induced voltage on the filtering inductors, with rotating transformation shifting it from 0 to $-j$. The zero from $v_{\text {odq }}$ to $i_{f d q}$ is the result of current-loop integral control which act as a virtual capacitor $C_{v}=1 / k_{i}$, combined with reactive current compensation control gain $j \omega C_{f}$ and current proportional control gain $k_{p}$. The zero from $v_{\text {odq }}$ to $i_{\text {odq }}$ is caused by $C_{v}$ and $C_{f}$ in parallel, so the total capacitor $C=C_{v}+C_{f}$.

TABLE I

TYPICAL PARAMETERS OF CVSC (PU).

\begin{tabular}{|c|c|c|c|}
\hline Parameter & Value & Parameter & Value \\
\hline$f_{s}$ & $12 \mathrm{kHz}$ & $a_{p}$ & 6.84 \\
\hline$C_{d c}$ & 9.30 & $a_{i}$ & 1.37 \\
\hline$L_{f}$ & 0.0498 & $b_{p}$ & 0 \\
\hline$C_{f}$ & 0.120 & $b_{i}$ & 0.400 \\
\hline$L_{o}$ & 0.0201 & $\kappa_{p}$ & 0.200 \\
\hline$k_{p}$ & 1.19 & $\kappa_{i}$ & 0.010 \\
\hline$k_{i}$ & 7.17 & & \\
\hline
\end{tabular}

TABLE II

DOMINANT POLES AND ZEROS OF FAST SUB-MODEL FOR CVSC.

\begin{tabular}{|c|c|c|c|c|}
\multirow{2}{*}{ output } & \multicolumn{2}{|c|}{$i_{d q}^{*}$} & \multicolumn{2}{c|}{$v_{\text {odq }}$} \\
\cline { 2 - 5 } & pole & zero & pole & zero \\
\hline$v_{c d q}$ & & $-j$ & & \\
\hline$i_{f d q}$ & & & & $-0.12+j 0.85$ \\
\hline$v_{f d q}$ & & $-j$ & & \\
\hline$i_{\text {odq }}$ & & & & 0 \\
\hline
\end{tabular}

TABLE III

SYMBOLIC TRANSFER FUNCTION MATRIX OF TRUNCATED FAST SUB-MODEL FOR CVSC.

\begin{tabular}{|c|c|c|}
\hline output & $i_{d q}^{*}$ & $v_{o d q}$ \\
\hline$v_{c d q}$ & $(s+j \omega)\left(L_{f}+L_{o}-\omega^{2} L_{o} L_{f} C_{f}\right)$ & $1-\omega^{2} L_{f} C_{f}$ \\
\hline$i_{f d q}$ & $1-\omega^{2} L_{o} C_{f}$ & $-s\left(1-j \omega C_{f} k_{p}\right)+j \omega C_{f}$ \\
\hline$v_{f d q}$ & $(s+j \omega) L_{o}$ & 1 \\
\hline$i_{o d q}$ & 1 & $-s\left(C_{v}+C_{f}\right)$ \\
\hline
\end{tabular}

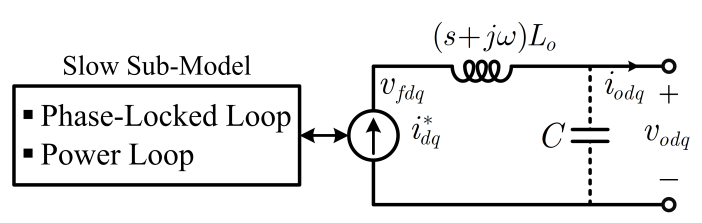

Fig. 7. Reduced-order equivalent circuit for CVSC according to Table III.

An equivalent circuit can be derived according to the transfer functions, as shown in Fig. 7. The capacitor $C$ can be further truncated when a CVSC is connected to a voltage source in a grid (see Section IV), so it is shown with dashed connection. Note that the current source $i_{d q}^{*}$ is in series with an inductor, which is not allowed in standard circuit theory. However, in this case, $i_{d q}^{*}$ comes from outer control loops and is differentiable in time, so it will not induce infinite voltage on the inductor. Moreover, $i_{d q}^{*}$ is a state variable in the slow submodel, so the series inductor does not generate extra states. As a result, the 8-order fast sub-model is truncated to 0 -order, 
but its essential information is shifted to the slow sub-model rather than discarded, since the time-variation of $i_{d q}^{*}$ induces an inductor voltage which affects power and phase angle in the slow sub-model.

\section{B. VVSC}

A VVSC has a different model from a CVSC, as shown in Fig. 8. In the slow sub-model, frequency-droop control is used instead of dc-link voltage control for power loop, and the phase-locked loop is omitted due to the self-synchronization characteristic of frequency droop regulation. In the fast submodel, a voltage loop is added to maintain terminal voltage actively. A virtual impedance $R_{v}+j X_{v}$ is also included to ensure that the total impedance is inductive, which is helpful for active and reactive power sharing, especially dynamically [31].

Following the proposed model reduction procedure, the typical parameters, the dominant poles and zeros, the truncated transfer functions, and the equivalent circuit diagram can be derived and are shown in Table IV, Table V, Table VI and Fig. 9 respectively. Again, the dominant zeros are caused by the induced voltage on inductors, but there is also a dominant pole which is absent in a CVSC. This pole comes from the combined effect of the output inductor $L_{o}$ and a virtual inductor $L_{v}$ caused by voltage control $\left(L_{v} \approx h_{v}^{-1}\right)$. Detailed interpretation of this pole is interesting but is not the focus of this paper, so is placed in the appendix. It turned out that this pole has significant impact on the system-wide dynamics and stability, which will be illustrated in the next section. To reflect this dominant pole, we peel off the 10-order fast sub-model but add back a 2-order sub-model in the reduced model.

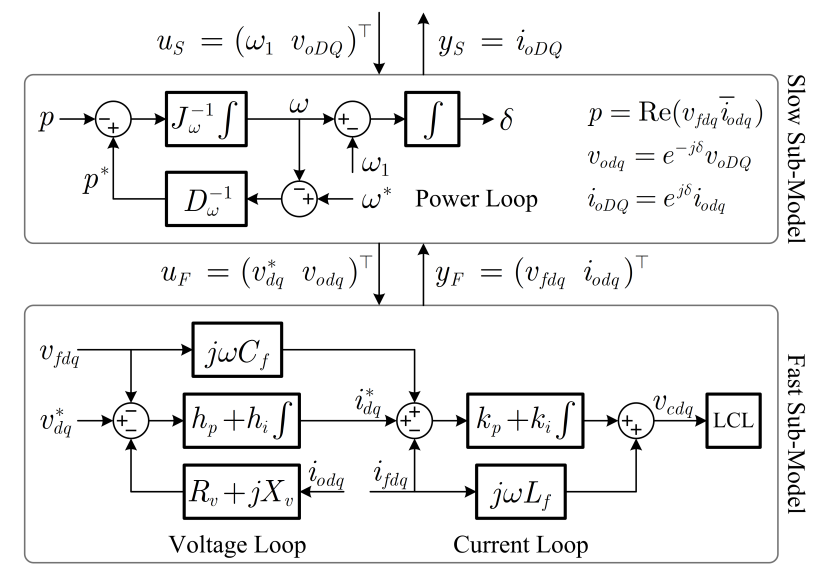

Fig. 8. VVSC model with power loop (droop), current loop, and LCL filter.

TABLE IV

TYPICAL PARAMETERS OF VVSC (PU).

\begin{tabular}{|c|c|c|c|}
\hline Parameter & Value & Parameter & Value \\
\hline$f_{s}$ & $8 \mathrm{kHz}$ & $h_{p}$ & 0.357 \\
\hline$L_{f}$ & 0.0292 & $h_{i}$ & 17.1 \\
\hline$C_{f}$ & 0.228 & $R_{v}$ & 0.01 \\
\hline$L_{o}$ & 0.0076 & $X_{v}$ & 0.0424 \\
\hline$k_{p}$ & 0.701 & $J_{\omega}$ & 500 \\
\hline$k_{i}$ & 4.21 & $D_{\omega}$ & 0.01 \\
\hline
\end{tabular}

TABLE $\mathrm{V}$

DOMINANT POLES AND ZEROS OF FAST SUB-MODEL FOR VVSC.

\begin{tabular}{|c|c|c|c|c|}
\hline \multirow{2}{*}{ output } & \multicolumn{2}{|c|}{$v_{d q}^{*}$} & \multicolumn{2}{c|}{$v_{\text {odq }}$} \\
\cline { 2 - 5 } & pole & zero & pole & zero \\
\hline$v_{f d q}$ & $-0.15-j 0.76$ & $-j$ & $-0.15-j 0.76$ & $-0.17-j 0.73$ \\
\hline$i_{\text {odq }}$ & $-0.15-j 0.76$ & & $-0.15-j 0.76$ & \\
\hline
\end{tabular}

TABLE VI

SYMBOLIC TRANSFER FUNCTION MATRIX OF TRUNCATED FAST SUB-MODEL FOR VVSC.

\begin{tabular}{|c|c|c|}
\hline output & $v_{d q}^{*}$ & $v_{o d q}$ \\
\hline$v_{f d q}$ & $(s+j \omega) L_{o} /(s L+j X+R)$ & $\left(s L_{v}+j X_{v}+R_{v}\right) /(s L+$ \\
& & $j X+R)$ \\
\hline$i_{o d q}$ & $1 /(s L+j X+R)$ & $-1 /(s L+j X+R)$ \\
\hline
\end{tabular}

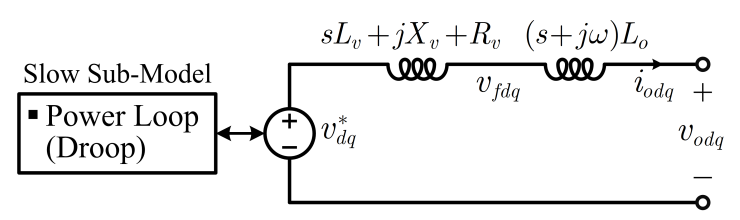

Fig. 9. Reduced-order equivalent circuit for VVSC according to Table VI.

It is worth noting that the reduced VVSC model is quite similar to a synchronous machine, except that the output resistance, reactance and inductance are determined not only by physical circuits $\left(L_{o}\right)$ but also by control parameters $\left(L_{v}\right.$, $X_{v}, R_{v}$ ). This certainly offers more flexibility in design, but care has to be taken to ensure that the control parameters are well-chosen, which will be discussed in the next section as well.

\section{Power System Stability}

Now that we have the reduced model for both CVSCs and VVSCs, we are ready to interconnect them in power systems for stability studies. As discussed in Section II, interconnection may generate new fast dynamics which need to be dealt with. In our case, when a CVSC is connected with a VVSC or other voltage sources, poles appears due to LC resonance, as shown in Fig. 10. These poles can be found from the following characteristic equation

$$
L_{2} C_{1} s^{2}+\left(j X_{2}+R_{2}\right) C_{1} s+1=0 .
$$

Using the parameters in the previous section, we get $s_{1}=$ $-0.08-j 8.0$ and $s_{2}=-0.07+j 7.3$, which are in highfrequency range. Since only low-frequency poles and zeros are preserved in the reduced models, it implies that the new high-frequency ones are generated by interactions arising from the interconnection. This mode can be poorly damped if $R_{2}$ is small, which is misleading since current-loop proportional control provides extra damping but is excluded in the truncated model. This problem can be fixed by performing another highfrequency truncation after interconnection, which is equivalent to omitting capacitor $C_{1}$. As a result, the reduced model for a CVSC is further simplified. 


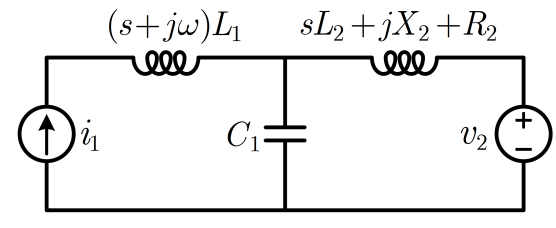

Fig. 10. Interconnection of CVSCs and VVSCs induces LC resonance.

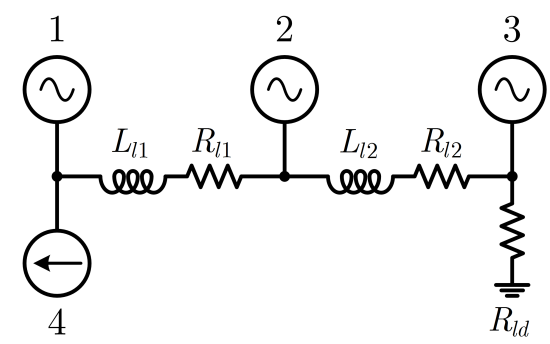

Fig. 11. The power system used for evaluation - a four terminal microgrid.

TABLE VII

NETWORK AND PASSIVE LOAD PARAMETERS (PU).

\begin{tabular}{|c|c|c|c|}
\hline Parameter & Value & Parameter & Value \\
\hline$L_{l 1}$ & 0.0262 & $L_{l 2}$ & 0.0262 \\
\hline$R_{l 1}$ & 0.0103 & $R_{l 2}$ & 0.0358 \\
\hline$R_{l d}$ & 2.0 & & \\
\hline
\end{tabular}

Finally, a mini power system was used as a case study to evaluate the accuracy of our reduced model in stability analysis. The evaluation system is a stand-alone microgrid consisting of three VVSCs and one CVSC, as depicted in Fig. 11. The VVSCs serve as sources with the intrinsic ability of maintaining voltage and frequency, whereas the CVSC serves as non-linear active load with constant power behaviour. This combination is representative of possible applications of model reduction since it contains most of the basic elements of a converter-rich power system, and allows observation of typical interaction patterns therein. The VSCs in the microgrid use the same parameters as those listed in Table I and Table IV, while the network parameters, appropriate to low voltage distribution lines, are presented in Table VII.

To quantify the accuracy of our reduced model, we compared the full and reduced model of the whole microgrid via eigen analysis [30], with results displayed in Fig. 12. The reduced model with the proposed "peel-off and add-back" methodology matches the low-frequency eigenvalues very well, whereas the one with merely "peel-off" has significant errors in several eigenvalues marked by the dashed boundary.

A further participation analysis reveals the nature of these modes and errors. Mode 1 is a swing mode between $\mathrm{VSC}_{1}$ and $\mathrm{VSC}_{3}$ since its main participation is by $\omega_{1}, \omega_{3}$ and $\delta_{3}$, as shown in Fig. 13. That said, the integrator current $i_{i d q}$ and output current $i_{\text {odq }}$ of $\mathrm{VSC}_{1,3}$ also have noticeable participation in this mode. As explained in Section IV, the integrator $h_{i}$ serves as a virtual inductor $L_{v}$, so $i_{i d q}$ is actually the current through $L_{v}$. Mode 2 is another swing mode between $\mathrm{VSC}_{1}$ and $\mathrm{VSC}_{2}$ and has similar participation. Mode 3 is a phase-locked loop mode and its main participation is by phase-locking states $\delta, \epsilon$ and $\omega$ of $\mathrm{VSC}_{4}$, as shown in Fig. 13(b). Again, the integrator and output current have noticeable participation, but only on $q$ axis $\left(i_{i q}\right.$ and $\left.i_{o q}\right)$. This is because only the $q$-axis voltage $v_{f q}$ is used for the phase-locked loop. In contrast, mode 4 is a dclink mode (Fig. 13(c)), in which only the $d$-axis currents have a noticeable participation, because dc-link voltage is closely related to active power which is set, in the main, by $v_{d}$ and $i_{d}$.
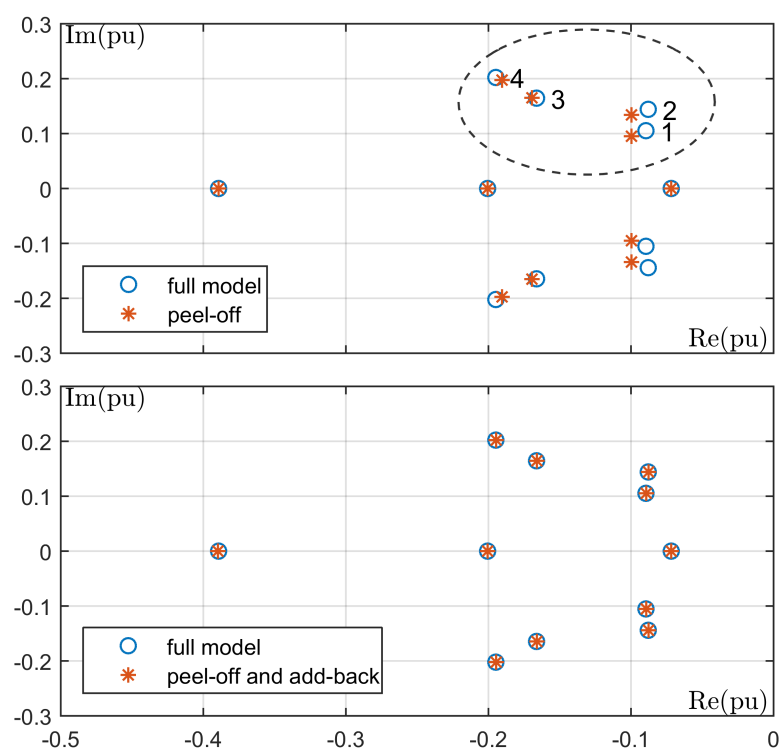

Fig. 12. Eigenvalues comparison. Top: reduced model with peel-off v.s. full model. Bottom: reduced model with peel-off and add-back v.s. full model.

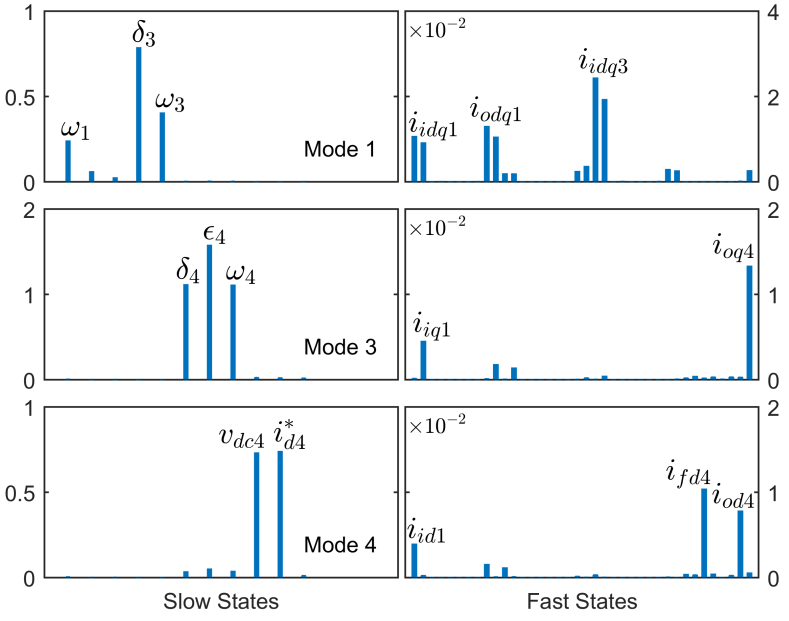

Fig. 13. Participation of error modes (mode 1,2 and 4).

The states $i_{i d q}, i_{f d q}$ and $i_{o d q}$ are all in the fast submodel and therefore omitted during peel-off. However, their participation in modes 1-4 indicates a non-trivial interaction exists and this explains why the peel-off method generates significant errors. On the other hand, the dominant parts of these states are restored via the add-back process and so the corresponding eigenvalues are then very well matched.

One may argue that the errors in Fig. 12 are still acceptable, but this is no longer true when some parameters are pushed 
to their extremes. In the case shown in Fig. 14, the droop coefficient $D_{\omega}$ was changed from 0.01 to 0.1 to make the swing modes unstable, but the peel-off model is seen to fail to predict this and misleadingly show the modes as stable. In contrast, the "peel-off and add-back" model closely matches the full model in this extreme case and shows the modes as unstable.
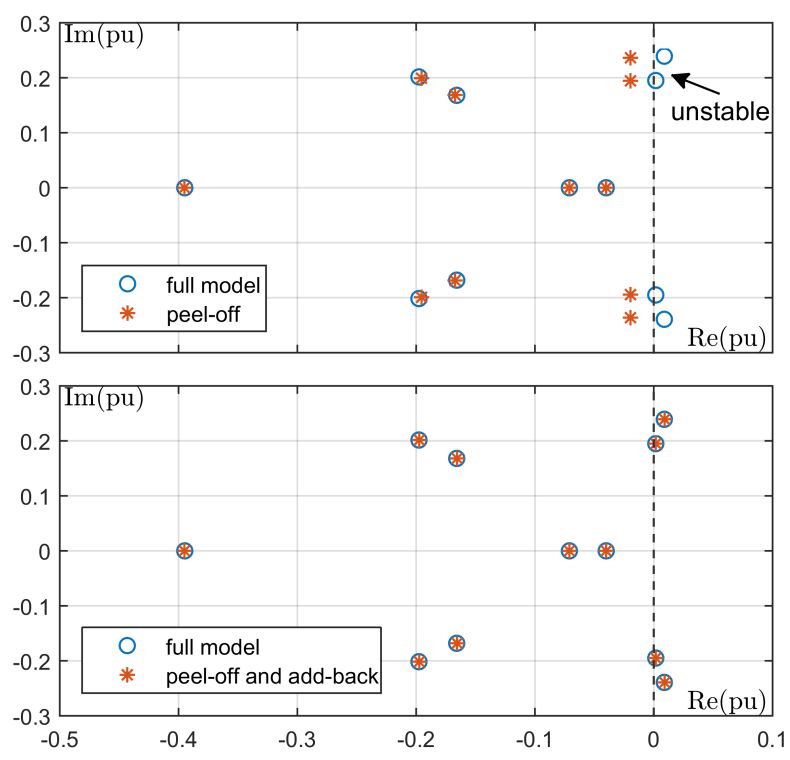

Fig. 14. Unstable eigenvalues. Top: reduced model with peel-off v.s. full model. Bottom: reduced model with peel-off and add-back v.s. full model.

The accuracy of the proposed model reduction methodology was tested under parameter variations. In Fig. 15, the inner loop control parameters $\left(k_{p}, k_{i}, h_{p}, h_{i}\right)$ and outer loop control parameters $\left(a_{p}, a_{i}, b_{p}, b_{i}, \kappa_{p}, \kappa_{i}, J_{\omega}\right)$ were changed and the corresponding model reduction error plotted

$$
e_{n}=\left|\left(\lambda_{n}-\lambda_{n}^{\prime}\right) / \lambda_{n}\right| \text {. }
$$

It is apparent that decreasing the inner loop gain or increasing the outer loop gain may lead to a larger error. This is natural since such variations bring the timescales of the fast and slow states closer together and cause greater interaction. Nonetheless, all errors are still kept below 1\% under large variations of gain $( \pm 10 \mathrm{~dB})$, which indicates that the model reduction method is accurate under a broad set of conditions.

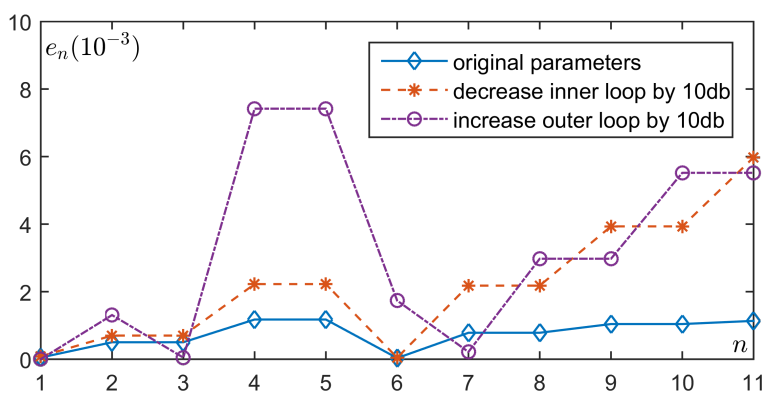

Fig. 15. Sensitivity of modelling errors to parameter variations.

Although the case study above is on a pure converter system, the proposed model reduction methodology is equally applicable to hybrid mechanical-electrical-electronic systems, due to its theoretic generality. To demonstrate this, we replace VSC1 in Fig. 11 by a synchronous generator (SG) of the same power rating, redo the model reduction, and exhibit the resultant eigenvalues in Fig. 16, which are well matched for "peel-off and add-back" method (the lower part of the figure). The inertia of a synchronous generator is higher than the virtual inertia of a VVSC, and this difference is reflected by the swing mode of the SG in the dashed boundary, which shows lower frequency than that of the swing mode of VVSCs marked by the arrows. Consequently, the conventional peel-off method has higher modelling error for the VVSC mode than for the SG mode, as shown in the upper part of Fig. 16, which supports our argument that the poor separation of timescale may cause poor accuracy in model reduction, and justifies the necessity of using the proposed "peel-off and add-back" method to account for high converter penetration.
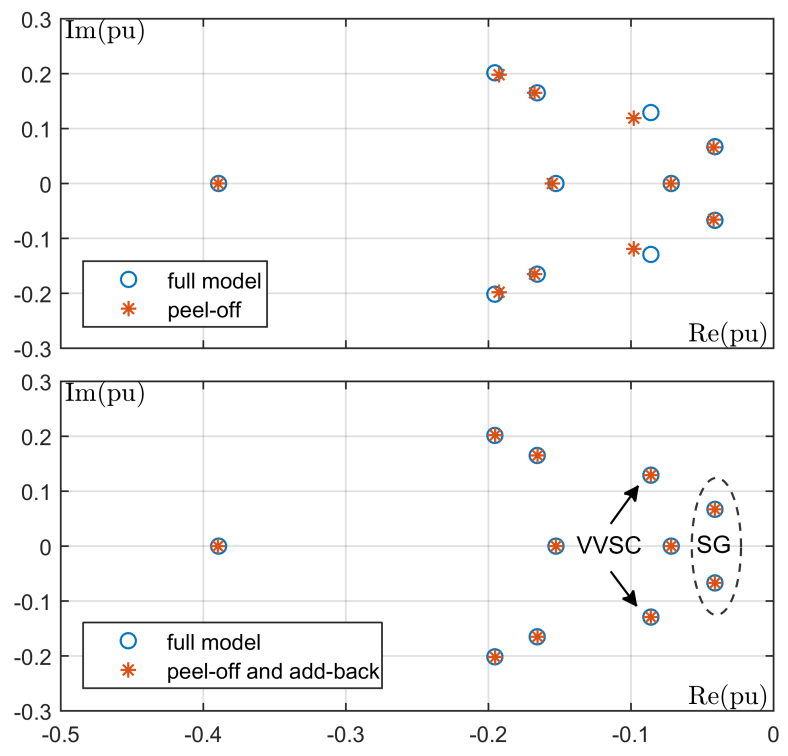

Fig. 16. Eigenvalues for a microgrid with a synchronous generator. Top: reduced model with peel-off v.s. full model. Bottom: reduced model with peel-off and add-back v.s. full model.

Having established the accuracy of the reduced model, attention is turned to the insights it can bring in control design, especially for the control functions that lie between fast and slow states. For example and as noted in Section III B, the dominant pole of the fast sub-model of a VVSC is

$$
z_{p}=-\left(R_{v}+j X_{v}+j \omega L_{o}\right)\left(L_{o}+L_{v}\right)^{-1}
$$

in which $L_{v}=h_{i}^{-1}$ is determined by voltage loop integral gain. This implies that voltage control may have significant influence on the slow dynamics. When $h_{i}$ is reduced, $z_{p}$ will be pushed further toward low-frequency range, resulting in stronger interaction between $z_{p}$ and slow states, which may affect stability. This observation is confirmed by Fig. 17, where it can be seen that the swing modes tend to be unstable with lower $h_{i}$ (a change of $-10 \mathrm{~dB}$ here). On the other hand, it has been shown [32] that higher voltage loop gain may cause stability problems for fast states by exciting resonance in LCL filter. Therefore, the selection of voltage loop gain is an art of 
trading-off between the stability of fast and slow dynamics. Previous research on voltage control has mainly focused on the fast dynamics, and its influence on slow modes is the new insight provided by the reduced model.

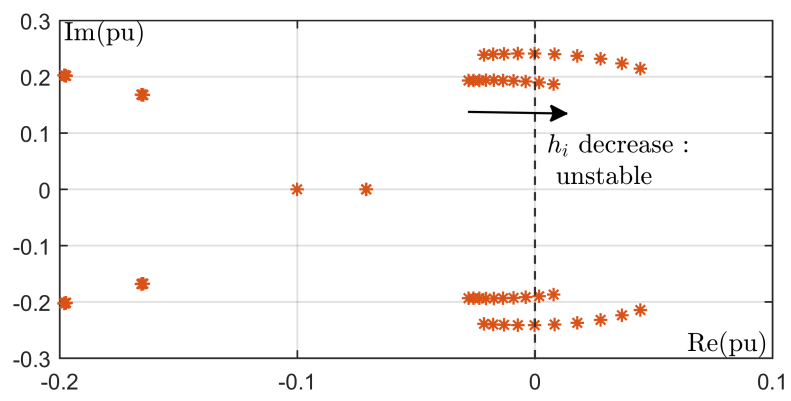

Fig. 17. Swing modes with reduced voltage loop gains.

\section{EXPERIMENTS}

A series of experiments were conducted for further verification of the model reduction. The experimental setup follows exactly the microgrid described in Section IV. Fig. 18, Fig. 19, and Fig. 20 give comparison of transient responses measured from experiments and generated by the reduced model, which match very well.

Fig. 21 displays the unstable case in which $D_{\omega}$ is increased from 0.01 to 0.1 . It is clear that there are two unstable oscillatory modes as predicted by the right-half-plane eigenvalues in Fig. 14(b). Note that the conventional peel-off model fails to identify this instability. These results show that the reduced model is accurate in terms of both small-signal and transient response, even in extreme scenarios.

Simulation results also show an accurate matching for the case with VSC1 replaced by a synchronous generator, verifying the general applicability of the proposed method on mechanical-electrical-electronic systems.

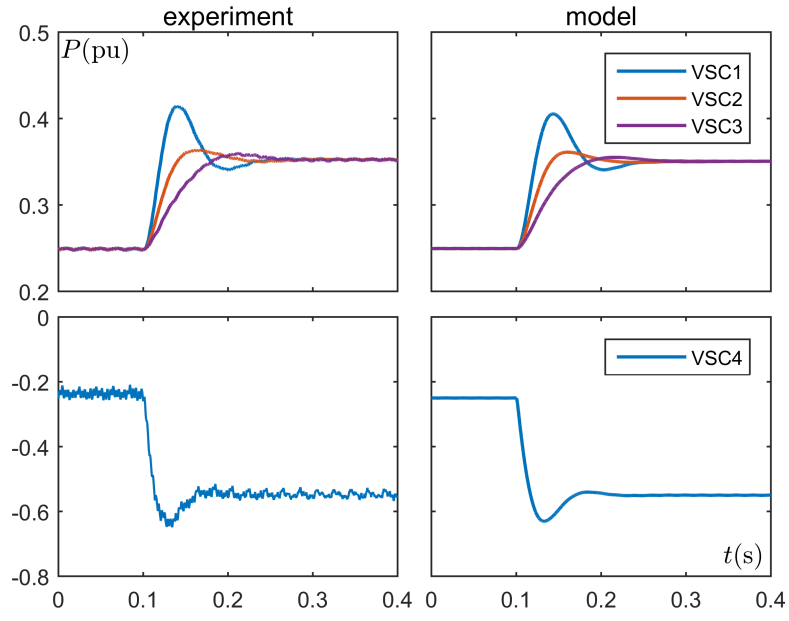

Fig. 18. Active power response (filtered) for VSC1-4 in load step change.
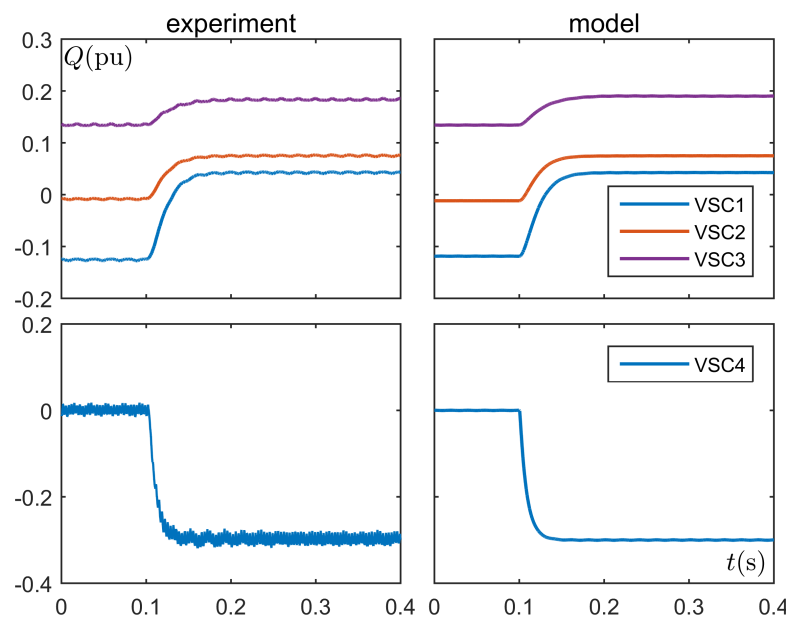

Fig. 19. Reactive power response (filtered) for VSC1-4 in load step change.
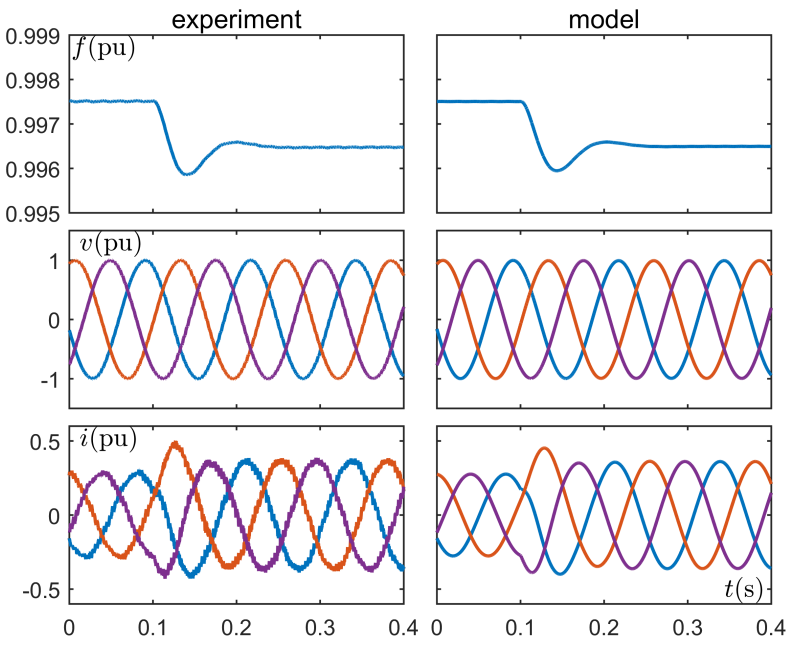

Fig. 20. Frequency, ac voltage and current for VSC1 in load step change.

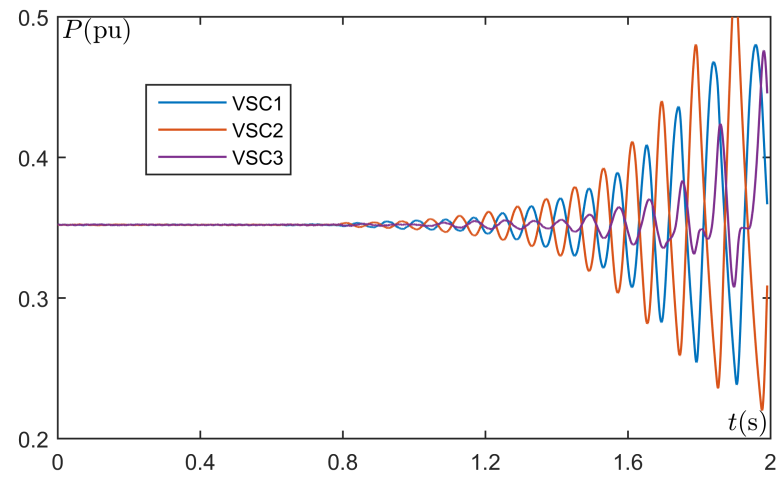

Fig. 21. Unstable case experiment result.

\section{CONCLUSION}

The model reduction methodology introduced in this paper proves to be an effective tool for simplifying the study of the stability of power systems rich in power converters. It is not simply a discarding of fast states but is a "peel-off 
and add-back" approach to restore the participation of some fast states in slow modes for systems where the separation in timescale is not complete. Add-back refers to adding a representation of the dominant part of the fast dynamics. Based on this concept, dominant modes were identified associated with the fast states for typical converters, which turn out to have noticeable interaction with slow states in the droop controllers, dc-link and phase-locked loops. It has been demonstrated that neglect of these interactions leads to inaccuracy in identifying the frequency and damping of some modes, and even misidentification of unstable modes as stable. Based on the reduced model, new design guidelines are also put forward for converter control. The efficacy of the proposed model reduction has been demonstrated through comparison of experimental and analytical results for a microgrid contain both voltage-controlled and current-controlled VSCs.

\section{APPENDIX A}

\section{A. Per-unit System}

A per-unit system is used to normalize all quantities to the corresponding base values in Table A.I, except where specially declared. These base values are also the rated values of all converters.

TABLE A.I

BASE VALUES FOR PER-UNIT SYSTEM.

\begin{tabular}{|c|c|c|}
\hline Base & Symbol & Value \\
\hline Power & $P_{b}$ & $10 \mathrm{~kW}$ \\
\hline Voltage & $V_{b}$ & $380 \mathrm{~V}$ \\
\hline Frequency & $f_{b}$ & $50 \mathrm{~Hz}$ \\
\hline Current & $I_{b}$ & $P_{b} / I_{b}$ \\
\hline Impedance & $Z_{b}$ & $V_{b} / I_{b}$ \\
\hline Admittance & $Y_{b}$ & $1 / Z_{b}$ \\
\hline Inductance & $L_{b}$ & $Z_{b} / 2 \pi f_{b}$ \\
\hline Capacitance & $C_{b}$ & $Y_{b} / 2 \pi f_{b}$ \\
\hline
\end{tabular}

\section{B. Model Reduction Calculation}

To simplify the calculation, we rewrite $G$ in polynomial fraction form

$$
G=N / D
$$

and perform truncation for $N$ and $D$ separately.

For a CVSC, $N_{C}$ and $D_{C}$ are shown in (A.2) and (A.3) at the top of the next page. It is clear that they are polynomials of less than order five so the solution is straightforward. Persevering all low-frequency roots while eliminating highfrequency ones, the transfer functions are reduced to $N_{C}^{\prime}$ and $D_{C}^{\prime}$

$$
\begin{gathered}
N^{\prime}{ }_{C}=k_{i}\left[\begin{array}{cc}
(s+j \omega)\left(L_{f}+L_{o}\right) & 1-\omega^{2} L_{f} C_{f} \\
1-\omega^{2} L_{o} C_{f} & s\left(j \omega C_{f} k_{p}-1\right) k_{i}^{-1}+j \omega C_{f} \\
(s+j \omega) L_{o} & 1 \\
1 & -s\left(C_{f}+k_{i}^{-1}\right)
\end{array}\right] \\
D_{C}^{\prime}=k_{i} .
\end{gathered}
$$

Letting $G_{C}^{\prime}=N_{C}^{\prime} / D_{C}^{\prime}$, we get Table III. Note that $k_{i}^{-1}=C_{v}$ acts as a virtual capacitor.

For a VVSC, $N_{V}$ and $D_{V}$ are shown in (A.6) and (A.7) at the top of the next page. $D_{V}$ is a fifth order polynomial which does not have a radical solution generally. A numerical solution may be used instead, but it is not as powerful as a symbolic one in relating to physical meanings. We manage to obtain an approximate symbolic solution below. The equation $D_{V}=0$ is rewritten as

$$
R_{v}+j X_{v}+j \omega L_{o}+s L_{o}+\frac{s D_{C}}{\left(s k_{p}+k_{i}\right)\left(s h_{p}+h_{i}\right)}=0 .
$$

$D_{C}$ and $\left(s k_{p}+k_{i}\right)\left(s h_{p}+h_{i}\right)$ only have high-frequency roots, which means they are insensitive to $s$ when $s$ is small. In fact, for a transfer function

$$
G(s)=\prod_{l=1}^{M}\left(s-z_{l}\right) / \prod_{k=1}^{N}\left(s-p_{k}\right)
$$

its relative sensitivity

$$
\left|\frac{\partial G}{\partial s} \frac{s}{G}\right|=\left|\sum_{l=1}^{M} \frac{s}{s-z_{l}}-\sum_{k=1}^{N} \frac{s}{s-p_{k}}\right| \ll 1
$$

if

$$
|s| \ll \min \left(\left|z_{l}\right|,\left|p_{k}\right|\right) /(M+N) .
$$

Therefore, we may replace their $s$ by 0 in solving the lowfrequency roots without losing much accuracy, and the equation becomes

$$
R_{v}+j X_{v}+j \omega L_{o}+s L_{o}+s h_{i}^{-1}=0 .
$$

Once the low-frequency root is solved, we can perform truncation to get $N_{V}^{\prime}$ and $D_{V}^{\prime}$

$$
\begin{gathered}
N^{\prime}{ }_{V}=\left[\begin{array}{cc}
(s+j \omega) L_{o} & s L_{v}+R_{v}+j X_{v} \\
1 & -1
\end{array}\right] \\
D^{\prime}{ }_{V}=s L_{v}+R_{v}+j X_{v}+s L_{o}+j \omega L_{o} .
\end{gathered}
$$

Letting $G_{V}^{\prime}=N_{V}^{\prime} / D_{V}^{\prime}$, and using the denotation below

$$
L_{v}=h_{i}^{-1}, L=L_{v}+L_{o}, X=X_{v}+\omega L_{o}, R=R_{v}
$$

we get Table VI.

(A.12) implies that the pole is not affected by the current control loop, since no current loop parameters appear in the equation. In contrast, the voltage loop has a strong influence on the dominant pole. In addition to the virtual impedance $X_{v}$ and $R_{v}$, the integral control gain $h_{i}$ serves as a virtual inductor $L_{v}$, which is in series with the output inductor $L_{o} . X_{v}, R_{v}$, $L_{v}$ and $L_{o}$ determine the dominant pole together.

Putting back the truncated sub-models, we finally get the reduced state-space models.

1) $\mathrm{CVSC}$ :

$$
\dot{x}=\left[\begin{array}{c}
\dot{\delta} \\
\varepsilon \\
\omega \\
v_{d c} \\
i_{d}^{*} \\
i_{q}^{*}
\end{array}\right]=\left[\begin{array}{c}
\omega-\omega_{(1)} \\
\left(v_{f q}-\varepsilon\right) \kappa_{\varepsilon} \\
v_{f q} \kappa_{\varepsilon} \kappa_{p}+\varepsilon\left(\kappa_{i}-\kappa_{\varepsilon} \kappa_{p}\right) \\
\left(p_{s}-p\right) v_{d c}^{-1} C_{d c}^{-1} \\
\left(v_{d c}-v_{d c}^{*}\right) a_{i}+\left(p_{s}-p\right) v_{d c}^{-1} C_{d c}^{-1} a_{p} \\
-\left(q^{*}-q\right) b_{i}
\end{array}\right]
$$




$$
\begin{aligned}
& N_{C}=\left[\begin{array}{cc}
(s+j \omega)\left((s+j \omega)^{2} L_{f} C_{f} L_{o}+L_{f}+L_{o}\right)\left(s k_{p}+k_{i}\right) & \left(j \omega(s+j \omega) L_{f} C_{f}+1\right)\left(s k_{p}+k_{i}\right)-j \omega s L_{f} \\
\left((s+j \omega)^{2} C_{f} L_{o}+1\right)\left(s k_{p}+k_{i}\right) & s\left(j \omega C_{f} k_{p}-1\right)+j \omega C_{f} k_{i} \\
(s+j \omega) L_{o}\left(s k_{p}+k_{i}\right) & s^{2} L_{f}+s k_{p}+k_{i} \\
s k_{p}+k_{i} & -s\left(s(s+j \omega) L_{f} C_{f}+C_{f}\left(s k_{p}+k_{i}\right)+1\right)
\end{array}\right] \\
& D_{C}=s^{2}\left((s+j \omega)^{2} L_{f} C_{f} L_{o}+L_{f}+L_{o}\right)+j \omega s L_{o}+\left(L_{o} C_{f} s(s+j \omega)+1\right)\left(s k_{p}+k_{i}\right) \\
& N_{V}=\left[\begin{array}{cc}
(s+j \omega) L_{o}\left(s k_{p}+k_{i}\right)\left(s h_{p}+h_{i}\right) & s\left(s^{2} L_{f}+s k_{p}+k_{i}\right)+\left(R_{v}+j X_{v}\right)\left(s k_{p}+k_{i}\right)\left(s h_{p}+h_{i}\right) \\
\left(s k_{p}+k_{i}\right)\left(s h_{p}+h_{i}\right) & -s^{2}\left(s(s+j \omega) L_{f} C_{f}+C_{f}\left(s k_{p}+k_{i}\right)+1\right)-\left(s k_{p}+k_{i}\right)\left(s h_{p}+h_{i}\right)
\end{array}\right] h_{i}^{-1} k_{i}^{-1} \\
& D_{V}=\left(\left(R_{v}+j X_{v}+j \omega L_{o}+s L_{o}\right)\left(s k_{p}+k_{i}\right)\left(s h_{p}+h_{i}\right)+s D_{i}\right) h_{i}^{-1} k_{i}^{-1} \\
& {\left[\begin{array}{l}
p \\
q
\end{array}\right]=\left[\begin{array}{cc}
1+\left(v_{o q} \omega C_{f}-i_{d}^{*} k_{o}\right) a_{1} L_{e} & i_{q}^{*} a_{1} L_{o} \\
-\left(v_{o d} \omega C_{f}+i_{q}^{*} k_{o}\right) b_{1} L_{e} & 1-i_{d}^{*} b_{1} L_{o}
\end{array}\right]^{-\top} \cdot\left[\begin{array}{c}
\left(i_{d}^{*} v_{o d}+i_{q}^{*} v_{o q}\right)\left(k_{o} k_{f}+\omega^{2} L_{e} C_{f}\right)+\left(i_{q}^{*} b_{0}+i_{d}^{*} a_{0}\right) k_{o} L_{e}+\left(v_{o d} b_{0}-v_{o q} a_{0}\right) \omega L_{e} C_{f} \\
i_{d}^{*} v_{o q}-i_{q}^{*} v_{o d}+\left(i_{d}^{*} a_{0}-i_{q}^{*} a_{0}\right) L_{o}+\left(i_{d}^{* 2}+i_{q}^{* 2}\right) \omega L_{o}
\end{array}\right]} \\
& \begin{array}{l}
p=\left(i_{o d} v_{o d}+i_{o q} v_{o q}\right) L_{v} L^{-1}+\left(i_{o d} v_{d}^{*}+i_{o q} v_{q}^{*}\right) L_{o} L^{-1}-\left(i_{o d}^{2}+i_{o q}^{2}\right) R_{v} L_{o} L^{-1} \\
q=\left(i_{o d} v_{o q}-i_{o q} v_{o d}\right) L_{v} L^{-1}+\left(i_{o d} v_{q}^{*}-i_{o q} v_{d}^{*}\right) L_{o} L^{-1}-\left(i_{o d}^{2}+i_{o q}^{2}\right) X_{v} L_{o} L^{-1}
\end{array}
\end{aligned}
$$

in which

$$
\begin{aligned}
& v_{f q}=v_{o q}+\left(q-q^{*}\right) b_{i} L_{o}+i_{d}^{*} \omega L_{o} \\
& k_{o}=1-\omega^{2} L_{o} C_{f} \\
& k_{f}=1-\omega^{2} L_{f} C_{f} \\
& L_{e}=L_{f}+L_{o}-\omega^{2} L_{o} L_{f} C_{f} \\
& a_{0}=\left(v_{d c}-v_{d c}^{*}\right) a_{i}+p_{s} v_{d c}^{-1} C_{d c}^{-1} a_{p} \\
& a_{1}=-v_{d c}^{-1} C_{d c}^{-1} a_{p} \\
& b_{0}=-q^{*} b_{i} \\
& b_{1}=b_{i}
\end{aligned}
$$

and $p$ and $q$ are given in (A.18) at the top of this page.

2) VVSC:

$$
\dot{x}=\left[\begin{array}{c}
\dot{\delta} \\
\omega \\
i_{o d} \\
i_{o q}
\end{array}\right]=\left[\begin{array}{c}
\omega-\omega_{(1)} \\
\left(\left(\omega^{*}-\omega\right) D_{\omega}^{-1}-p\right) J_{\omega}^{-1} \\
\left(v_{d}^{*}-v_{o d}-i_{o d} R+i_{o q} X\right) L^{-1} \\
\left(v_{q}^{*}-v_{o q}-i_{o q} R-i_{o d} X\right) L^{-1}
\end{array}\right]
$$

in which $p$ and $q$ are given in (A.20) at the top of this page.

\section{REFERENCES}

[1] S. Rubenoff. (2016, Dec) Sungrow connects chinas largest solar plus storage microgrid project. [Online]. Available: https://microgridknowledge.com/microgrid-project-sungrow/

[2] D. Boroyevich, I. Cvetkovic, R. Burgos, and D. Dong, "Intergrid: A future electronic energy network?" IEEE J. Emerg. Sel. Topics Power Electron., vol. 1, no. 3, pp. 127-138, Sept. 2013.

[3] N. Flourentzou, V. G. Agelidis, and G. D. Demetriades, "VSC-based HVDC power transmission systems: An overview," IEEE Trans. Power Electron., vol. 24, no. 3, pp. 592-602, Mar. 2009.

[4] J. M. Carrasco, L. G. Franquelo, J. T. Bialasiewicz, E. Galvan, R. C. PortilloGuisado, M. A. M. Prats, J. I. Leon, and N. Moreno-Alfonso, "Power-electronic systems for the grid integration of renewable energy sources: A survey," IEEE Trans. Ind. Electron., vol. 53, no. 4, pp. 10021016, June 2006.

[5] M. E. Baran and N. R. Mahajan, "DC distribution for industrial systems: opportunities and challenges," IEEE Trans. Ind. Appl., vol. 39, no. 6, pp. 1596-1601, Nov.-Dec. 2003.

[6] E. Barklund, N. Pogaku, M. Prodanovic, C. Hernandez-Aramburo, and T. C. Green, "Energy management in autonomous microgrid using stability-constrained droop control of inverters," IEEE Trans. Power Electron., vol. 23, no. 5, pp. 2346-2352, Sept. 2008.
[7] L. P. Kunjumuhammed, B. C. Pal, C. Oates, and K. J. Dyke, "The adequacy of the present practice in dynamic aggregated modeling of wind farm systems," IEEE Transactions on Sustainable Energy, vol. 8, no. 1, pp. 23-32, Jan 2017.

[8] J. Sun, "Impedance-based stability criterion for grid-connected inverters," IEEE Trans. Power Electron., vol. 26, no. 11, pp. 3075-3078, Nov. 2011.

[9] J. Sun, "Small-signal methods for AC distributed power systems - A review," IEEE Trans. Power Electron., vol. 24, no. 11, pp. 2545-2554, Nov. 2009.

[10] X. Wang, F. Blaabjerg, and W. Wu, "Modeling and analysis of harmonic stability in an AC power-electronics-based power system," IEEE Trans. Power Electron., vol. 29, no. 12, pp. 6421-6432, Dec. 2014.

[11] M. Cheah-Mane, L. Sainz, J. Liang, N. Jenkins, and C. E. U. Loo, "Criterion for the electrical resonance stability of offshore wind power plants connected through hvdc links," IEEE Transactions on Power Systems, vol. PP, no. 99, pp. 1-1, 2017.

[12] X. Wang, L. Harnefors, F. Blaabjerg, and P. C. Loh, "A unified impedance model of voltage-source converters with phase-locked loop effect," in 2016 IEEE Energy Conversion Congress and Exposition (ECCE), Sept 2016, pp. 1-8.

[13] D. Pan, X. Ruan, X. Wang, H. Yu, and Z. Xing, "Analysis and design of current control schemes for lcl-type grid-connected inverter based on a general mathematical model," IEEE Transactions on Power Electronics, vol. 32, no. 6, pp. 4395-4410, June 2017.

[14] C. E. Ugalde-Loo, J. B. Ekanayake, and N. Jenkins, "State-space modeling of wind turbine generators for power system studies," IEEE Trans. Ind. Appl., vol. 49, no. 1, pp. 223-232, Jan.-Feb 2013.

[15] N. Kroutikova, C. a. Hernandez-Aramburo, and T. C. Green, "Statespace model of grid-connected inverters under current control mode," IET Electric Power Applications, vol. 1, no. 3, pp. 329-338, May 2007.

[16] J. Kevorkian and J. D. Cole, Multiple Scale and Singular Perturbation Methods. Springer, Sept. 2012, vol. 114.

[17] P. V. Kokotovic, R. O'malley, and P. Sannuti, "Singular perturbations and order reduction in control theory - An overview," Automatica, vol. 12, no. 2, pp. 123-132, Mar. 1976.

[18] K. Mease, S. Bharadwaj, and S. Iravanchy, "Timescale analysis for nonlinear dynamical systems," Journal of Guidance, Control, and Dynamics, vol. 26, no. 2, pp. 318-330, Mar.-Apr. 2003.

[19] P. Kundur, N. J. Balu, and M. G. Lauby, Power System Stability and Control. McGraw-hill, 1994, vol. 7.

[20] R. W. Erickson and D. Maksimovic, Fundamentals of Power Electronics. Springer Science, 2007.

[21] N. Bottrell, M. Prodanovic, and T. C. Green, "Dynamic stability of a microgrid with an active load," IEEE Trans. Power Electron., vol. 28, no. 11 , pp. 5107-5119, Nov. 2013.

[22] S. Gugercin and A. C. Antoulas, "A survey of model reduction by balanced truncation and some new results," International Journal of Control, vol. 77, no. 8, pp. 748-766, May 2004. 
[23] E. Davison, "A method for simplifying linear dynamic systems," IEEE Trans. Autom. Control, vol. 11, no. 1, pp. 93-101, Jan. 1966

[24] W. J. Rugh, Linear System Theory. Prentice Hall, 1996, vol. 2.

[25] H. Sandberg, E. Mollerstedt, and Bernhardsson, "Frequency-domain analysis of linear time-periodic systems," IEEE Transactions on Automatic Control, vol. 50, no. 12, pp. 1971-1983, Dec 2005.

[26] M. Guan and Z. Xu, "Modeling and control of a modular multilevel converter-based HVDC system under unbalanced grid conditions," IEEE Trans. Power Electron., vol. 27, no. 12, pp. 4858-4867, Dec. 2012.

[27] J. Peralta, H. Saad, S. Dennetiere, J. Mahseredjian, and S. Nguefeu, "Detailed and averaged models for a 401-level MMC-HVDC system," IEEE Trans. Power Del., vol. 27, no. 3, pp. 1501-1508, Jul. 2012.

[28] Q. Li and P. Wolfs, "A review of the single phase photovoltaic module integrated converter topologies with three different DC link configurations," IEEE Trans. Power Electron., vol. 23, no. 3, pp. 1320-1333, May 2008.

[29] J. Rocabert, A. Luna, F. Blaabjerg, and P. Rodrguez, "Control of power converters in AC microgrids," IEEE Trans. Power Electron., vol. 27, no. 11, pp. 4734-4749, Nov. 2012.

[30] N. Pogaku, M. Prodanovic, and T. C. Green, "Modeling, analysis and testing of autonomous operation of an inverter-based microgrid," IEEE Trans. Power Electron., vol. 22, no. 2, pp. 613-625, Mar. 2007.

[31] J. He and Y. W. Li, "Analysis, design, and implementation of virtual impedance for power electronics interfaced distributed generation," IEEE Trans. Ind. Appl., vol. 47, no. 6, pp. 2525-2538, Nov.-Dec. 2011.

[32] X. Wang, P. C. Loh, and F. Blaabjerg, "Stability analysis and controller synthesis for single-loop voltage-controlled VSIs," pp. 1-1, 2017.

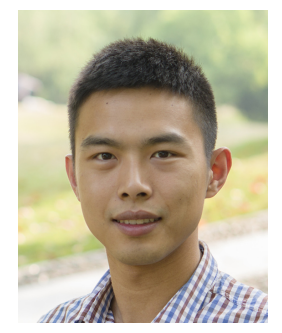

Yunjie Gu (S'14) received the B.Sc. and the Ph.D. degree in Electrical Engineering from Zhejiang University, Hangzhou, China, in 2010 and 2015 respectively. He was a Research Consultant at General Electric Global Research Centre, Shanghai, from 2015 to 2016, and is now a Research Associate at Imperial College, London.

His research interests include power system control and stability, and the application of power electronics to power systems.

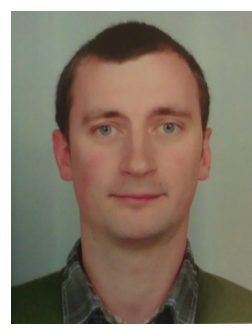

Nathaniel Bottrell (S'10) received the M.Eng. and the Ph.D. degree in Electrical and Electronic Engineering from Imperial College, London, UK., in 2009 and 2014 respectively, and is currently a Research Associate at Imperial College, London, UK.

His research interests include distributed generation, microgrids and the application of power electronics to low-voltage power systems.

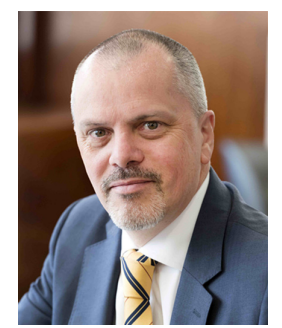

Timothy C. Green (M'89-SM'02) received a B.Sc. (Eng) (first class honours) from Imperial College London, UK in 1986 and a Ph.D. from Heriot-Watt University, Edinburgh, UK in 1990. He is a Professor of Electrical Power Engineering at Imperial College London, and Director of the Energy Futures Lab with a role fostering interdisciplinary energy research. He also leads the HubNet consortium of UK universities. His research interest is in using the flexibility of power electronics to accommodate new generation patterns and new forms of load, such as EV charging, as part of the emerging smart grid. In HVDC he has contributed converter designs that reduce losses while also providing control functions assist AC system integration. In distribution systems, he has pioneered the use of soft open points and the study of stability of grid connected inverters. 